\title{
Determinação do antígeno carcinoembrionário biliar na detecção das metástases hepáticas do carcinoma colorretal ${ }^{1}$
}

\author{
Adriana Polycarpo ${ }^{2}$, \\ Flávia Renata Topciư, \\ Julio Zaki Abucham Neto ${ }^{4}$, \\ Leonardo Seligra Lopes, \\ Luis Gustavo Coelho Catelani', \\ Maria Helena de Toledo Zerwes? \\ Sérgio Gonçalves ${ }^{8}$, \\ Jaques Waisberg 9
}

\begin{abstract}
Polycarpo A, Topciu FR, Neto JZA, Lopes LS, Catelani LGC, Zerwes MHT, Gonçalves S, Waisberg J. Determinação do antígeno carcinoembrionário (CEA) biliar na detecção das metástases hepáticas do carcinoma colorretal. Acta Cir Bras [online] 2003; 18 Supl 4. Disponível em www.scielo.br/acb.

RESUMO - Objetivo: Analisar, prospectivamente, os resultados da determinação do antígeno carcinoembriário (CEA) na bile vesicular, relacionando-os com os aspectos morfológicos e clínicos da neoplasia e recidiva hepática. Métodos: Os níveis do CEA foram estudados na bile vesicular e no sangue periférico de 44 doentes com carcinoma colorretal e 10 com colelitíase não complicada, a partir de amostras do CEA colhidas imediatamente antes da extirpação da neoplasia colo-retal e da colecistectomia (considerou-se valor normal até $5 \mathrm{ng} / \mathrm{ml}$ ). Resultados: Os 44 carcinomas colorretais extirpados com intenção curativa tiveram nível médio do CEA sérico de 8,5 ng/ml e CEA biliar, 74,5 ng/ml. Nas colelitíases não complicadas submetidas a colecistectomia, o nível médio do CEA sérico foi de $1,9 \mathrm{ng} / \mathrm{ml}$ e CEA biliar, 1,2 ng/ml. Quatro doentes submetidos à extirpação do carcinoma colo-retal, sem evidências de metástases hepáticas e com valor médio de CEA biliar de 213,2 ng/ml apresentaram metástases hepáticas entre três a 17 meses após a extirpação. Conclusão: o nível elevado de CEA biliar dos operados por carcinoma colo-retal pode indicar presença de metástases hepáticas e esses enfermos devem ser acompanhados com especial atenção para diagnosticar essas lesões.
\end{abstract}

DESCRITORES - Antígeno carcinoembrionário. Neoplasias colorretais. Metástase neoplásica.

\section{Introdução}

O câncer colo-retal é o segundo mais freqüente no mundo ocidental e o fígado é o órgão mais acometido por suas metástases. ${ }^{1,2}$ No momento da extirpação do tumor primário, metástases hepáticas são encontradas em 20 a $25 \%$ dos doentes e, aproximadamente, metade dos enfermos que tiveram suas lesões colorretais extirpadas de maneira aparentemente curativa desenvolverá lesões hepáticas no pós-operatório. . $^{1,3,4}$
A maioria dos programas de monitorização da recidiva do carcinoma colorretal operado incluem, em intervalos regulares, a determinação da concentração sérica do antígeno carcinoembrionário (CEA) e imagens hepáticas por ultrasonografia, tomografia e/ou ressonância magnética abdominais. ${ }^{3,5}$ Entretanto, mesmo com tal investigação, cerca de 10 a $30 \%$ das metástases hepáticas permanecerão não diagnosticadas. ${ }^{4}$ Quando presentes, a extirpação das lesões no fígado é o tratamento de

1. Trabalho realizado no Departamento de Gastroenterologia Cirúrgica do Hospital do Servidor Público Estadual de São Paulo - FMO. Vencedor na categoria cirúrgico do $28^{\circ}$. Congresso Médico Universitário do ABC.

2. Acadêmica de medicina da Faculdade de Medicina do ABC

3. Acadêmica de medicina da Faculdade de Medicina do ABC

4. Acadêmico de medicina da Faculdade de Medicina do ABC

5. Acadêmico de medicina da Faculdade de Medicina do ABC

6. Acadêmico de medicina da Faculdade de Medicina do ABC

7. Acadêmica de medicina da Faculdade de Medicina do ABC

8. Acadêmico de medicina da Faculdade de Medicina do ABC

9. Prof. Assistente da Disciplina de Cirurgia do Aparelho Digestivo da Faculdade de Medicina do ABC e Médico Assistente do Serviço de Gastroenterologia Cirúrgica do Hospital do Servidor Público Estadual de São Paulo - FMO. 
escolha, o que é possível em apenas $20 \%$ dos enfermos e somente $25 \%$ desses alcançarão sobrevivência maior que cinco anos..$^{1,3,4}$.

Gold e Freedman ${ }^{6}$ descreveram, em 1965, a presença do CEA em extratos de tumores malignos e no tecido intestinal de feto. Atualmente, a dosagem do CEA sangüíneo é utilizada no pré e pós-operatórios em portadores de carcinoma colorretal extirpado para a detecção da recidiva da doença. ${ }^{7-10}$

Em 1989, Yeatman e col. ${ }^{11}$ sugeriram que a concentração biliar do CEA poderia constituir marcador para detecção de metástases hepáticas em estádio mais precoce. Sua hipótese foi baseada nas observações de que o CEA derivado das metástases hepáticas pode ser excretado tanto na bile quanto no sangue. Como o volume biliar é menor que o plasmático, a concentração detectável de CEA eleva-se mais precoce na vesícula biliar do que no sangue periférico. ${ }^{11-13}$ Yeatman e col. ${ }^{11,12}$ e Paul e col ${ }^{13,14}$ encontraram elevadas concentrações biliares de CEA em doentes com carcinoma colorretal e metástases hepáticas comprovadas e em enfermos submetidos a extirpações curativas e sem envolvimento hepático detectado pelos métodos de imagem.

O papel do nível do CEA na bile da vesícula biliar permanece controverso em relação a sua contribuição para a detecção precoce de metástases hepáticas nos doentes operados curativamente de carcinoma colorretal. ${ }^{15-23}$

O objetivo deste estudo foi analisar prospectivamente, numa série de doentes operados curativamente de carcinoma colorretal, os resultados da determinação do CEA no sangue periférico e na bile da vesícula biliar, relacionando-os com os aspectos morfológicos da neoplasia e com a recidiva hepática.

\section{Métodos}

Entre 1998 e 2001, 44 doentes foram submetidos à extirpação de carcinoma colorretal com intenção curativa. O termo curativo foi utilizado para designar a ausência de doença macroscópica ao final do procedimento cirúrgico e pela verificação de laudo do exame anatomopatológico da lesão neoplásica extirpada e de estruturas associadas. Foram colhidas amostras de bile da vesícula biliar e do sangue periférico durante o ato operatório, imediatamente antes do início da exérese da neoplasia de todos enfermos.

Esta investigação foi realizada de acordo com os padrões éticos aceitos pela Declaração de Helsinski da Associação Médica Mundial, adotada em 1964 e emendada em 1996. Os doentes tiveram conhecimento do protocolo de estudo e assinaram Termo de consentimento Livre e Esclarecido ao ingresso na presente pesquisa.

Foram considerados critérios de inclusão a realização de operação curativa, ausência de metástases à distância e presença de adenocarcinoma do intestino grosso confirmada por meio do estudo histopatológico da lesão extirpada. Doentes submetidos a operações avaliadas como não curativas não foram incluídos nessa casuística.

Os dados clínicos e morfológicos foram obtidos por consulta aos prontuários hospitalares dos enfermos incluídos no estudo ou por entrevista com os doentes ou familiares nos retornos ambulatoriais.
Trinta e nove $(88,6 \%)$ enfermos eram brancos, três eram orientais $(6,8 \%)$ e dois $(4,5 \%)$ eram negros. Vinte e um doentes $(47,7 \%)$ eram do sexo masculino e $23(52,3 \%)$ do sexo feminino. A média de idade foi de $63 \pm 14,7$ anos (29 a 90 anos). Todos enfermos tiveram o diagnóstico no pré-operatório de carcinoma colo-retal confirmado por exame histológico de biópsias obtidas por colonoscopia, cujas lâminas foram coradas pelo método da hematoxilina - eosina (HE) e analisadas por patologista.

Considerou-se como Dukes $\mathrm{A}$, as neoplasias que não atingiam a túnica muscular externa da parede intestinal; como Dukes B, as que se estendiam por toda a parede, atingindo inclusive o tecido adiposo adventício e como Dukes $\mathrm{C}$, aquelas com comprometimento de linfonodos, independentemente da profundidade da invasão parietal.

A investigação pré-operatória da presença de lesão extraintestinal, realizada por ultra-sonografia abdominal, tomografia abdominal e radiografia de tórax, não revelou metástases em nenhum dos enfermos.

Em dez doentes portadores de colecistolitíase não complicada, submetidos a colecistectomia eletiva por laparotomia, foram colhidas amostras sangüíneas e da bile vesicular nas mesmas condições que a dos doentes portadores de carcinoma colorretal e serviram para fornecer os níveis de CEA na bile da vesícula biliar para comparação. Todos enfermos eram brancos, sendo sete $(70 \%)$ do sexo feminino e três $(30 \%)$ do sexo masculino. A média de idade foi de 50,8 \pm 20,1anos ( 23 a 74 anos).

As coletas das alíquotas sangüíneas e da bile foram realizadas durante o ato operatório. Após inventário da cavidade abdominal eram obtidas amostras de $5 \mathrm{ml}$ de bile da vesícula biliar pela punção da região fúndica da vesícula, por meio de agulha calibre 23, acoplada à seringa plástica de $10 \mathrm{ml}$ de capacidade. Em seguida, com outra seringa de $20 \mathrm{ml} \mathrm{de}$ capacidade, procedia-se ao esvaziamento do restante da bile da vesícula biliar, seguida pela oclusão do local de punção por sutura em bolsa com fio atraumático fino e absorvível de ácido poliglicólico. No início da operação, previamente à retirada da neoplasia colorretal, amostras sangüíneas de $5 \mathrm{ml}$ foram colhidas através de punção venosa periférica no membro superior não dominante e destituído de infusão intravenosa de qualquer solução, com agulha calibre 37 acoplada à seringa plástica de 10 $\mathrm{ml}$ de capacidade.

As amostras de soro e as amostras de bile, foram estocadas em congelador $\mathrm{a}-70^{\circ} \mathrm{C}$ até que as análises do CEA fossem realizadas. Para a dosagem dos CEA biliar e sérico foi utilizado ensaio fluoro-imunométrico de fase sólida (Delfia CEA Kit, Pharmacia, Turku-Finland). A precisão do método foi estimada por coeficiente de variação (c.v.) intra-ensaio de 3,4 e 2,4\% para valores baixos e altos, respectivamente, e o c.v. inter-ensaio foi de $4.6 \%$ e $2,8 \%$ para os mesmos parâmetros. A sensibilidade deste ensaio de CEA foi de $0,2 \mathrm{ng} / \mathrm{ml}$ e o ponto superior da curva de reconhecimento foi de $500 \mathrm{ng} / \mathrm{ml}$. Sempre que este valor foi ultrapassado, diluições foram necessárias para a adequação das reações.

Adotou-se o nível de normalidade do CEA biliar de até 5 $\mathrm{ng} / \mathrm{ml}$ baseados na análise dos valores obtidos no grupo de pacientes colecistectomizados. 
$\mathrm{Na}$ avaliação dos resultados utilizou-se testes estatísticos não paramétricos em função das amostras existentes. As variáveis quantitativas foram representadas por freqüência absoluta (N) e relativa (\%). Foram utilizados os modelos estatísticos da média aritmética, desvio padrão, teste de MannWhitney, teste de Wilcoxon e teste de Kruskal - Wallis. Foi testada a normalidade dos dados pelo teste de KolmogorovSmirnov e a homogeneidade da variância foi verificada pelo teste de Levene.

Em todos os testes, fixou-se em $0,05 \%$ o nível para a rejeição da hipótese de nulidade (nível de significância de 95\%) de acordo com os padrões correntes em estudos biológicos.

\section{Resultados}

No grupo de enfermos com carcinoma colorretal, a lesão foi única em $43(97,7 \%)$ doentes e múltipla em um $(2,3 \%)$ deles. A neoplasia localizava-se no reto em 24 (54,5\%) doentes, em seis $(13,7 \%)$ no ceco-ascendente, em outros seis $(13,6 \%)$ no colo transverso, em quatro $(9,1 \%)$ no sigmóide, em dois $(4,5 \%)$ no colo descendente, em um $(2,3 \%)$ na flexura esquerda e em um $(2,3 \%)$ a lesão envolvia o ceco e o reto simultaneamente. Em relação ao grau de diferenciação histopatológica da neoplasia, todas lesões foram consideradas moderadamente diferenciadas.

Todos doentes operados por carcinoma colorretal evoluíram sem intercorrências importantes e obtiveram alta hospitalar. A média do tempo de seguimento dos doentes foi de 16,5 meses ( 6 a 48 meses).

Em relação ao estadiamento pela classificação de Dukes, dois $(4,6 \%)$ enfermos foram classificados como Dukes A, 21 (47,7\%) como Dukes B e 21 (47,7\%) como Dukes C.

Treze $(29,5 \%)$ doentes classificados como Dukes $\mathrm{C}$ foram submetidos à quimioterapia adjuvante com 5-fluorouracil intravenoso por sete sessões.

Nos enfermos com carcinoma colorretal, o valor médio do CEA sérico foi de 8,5 $\pm 18,7 \mathrm{ng} / \mathrm{ml}(0,1 \mathrm{a} 111,0 \mathrm{ng} / \mathrm{ml})$ e o valor médio do CEA biliar foi de 74,5 $\pm 130,3(0,2$ a $571 \mathrm{ng} / \mathrm{ml})$ (Tabela 1).

TABELA 1 - Médias dos valores do CEA sérico e biliar obtidos dos doentes operados por carcinoma colorretal.

\begin{tabular}{c|c|c|c|c|c}
\hline Variável & Média & D.P. & Mínimo & Máximo & N \\
\hline CEA sérico & $8,5 \mathrm{ng} / \mathrm{ml}$ & 18,7 & $0,1 \mathrm{ng} / \mathrm{ml}$ & $111,0 \mathrm{ng} / \mathrm{ml}$ & 44 \\
\hline CEA biliar & $74,5 \mathrm{ng} / \mathrm{ml}$ & 130,26 & $0,2 \mathrm{ng} / \mathrm{ml}$ & $571,0 \mathrm{ng} / \mathrm{ml}$ & 44 \\
\hline
\end{tabular}

Legenda: Wilcoxon $(Z)=-4,614 \quad \mathrm{p}<0,0001 *$ D.P. $=$ Desvio padrão $\mathrm{N}=$ Número

No grupo de doentes com colelitíase não complicada, o valor médio do CEA sérico atingiu $1,94 \mathrm{ng} / \mathrm{ml} \pm 0,8(1,0$ a $3,5 \mathrm{ng} /$ $\mathrm{ml}$ ) e o valor médio do CEA biliar foi de 1,24 ng/ml $\pm 0,9$ (0,3 a 2,9 $\mathrm{ng} / \mathrm{ml}$ ) (Tabela 2). Os enfermos desse grupo obtiveram alta hospitalar sem intercorrências.
TABELA 2 - Médias dos valores do CEA sérico e biliar obtidos dos doentes operados por colelitíase não complicada.

\begin{tabular}{c|c|c|c|c|c}
\hline Variável & Média & D.P. & Mínimo & Máximo & $\mathbf{N}$ \\
\hline CEA sérico & $1,9 \mathrm{ng} / \mathrm{ml}$ & 0,8 & $1,0 \mathrm{ng} / \mathrm{ml}$ & $3,5 \mathrm{ng} / \mathrm{ml}$ & 10 \\
\hline CEA biliar & $1,2 \mathrm{ng} / \mathrm{ml}$ & 0,9 & $0,3 \mathrm{ng} / \mathrm{ml}$ & $2,9 \mathrm{ng} / \mathrm{ml}$ & 10 \\
\hline
\end{tabular}

Legenda: Wilcoxon $(Z)=-1,992$ p $<0,46 *$ D.P.= Desvio padrão $\mathrm{N}=$ Número

Dezessete $(38,6 \%)$ doentes apresentaram valor sérico do CEA acima de 5,0 ng/ml, enquanto $27(61,4 \%)$ enfermos exibiram nível sangüíneo do CEA menor ou igual a $5,0 \mathrm{ng} / \mathrm{ml}$. Em relação ao CEA biliar, $29(65,9 \%)$ doentes exibiram valores acima de 5,0 $\mathrm{ng} / \mathrm{ml}$, ao passo que em $15(34,1 \%)$ sua determinação estava igual ou inferior a 5,0 ng/ml. Em 35 (79,5\%) enfermos, o nível do CEA biliar era maior que o do CEA sérico e em nove $(20,5 \%)$ o valor do CEA sérico foi mais elevado que o do CEA biliar. Treze $(29,5 \%)$ doentes apresentaram, simultaneamente, valores do CEA sérico e do CEA biliar acima de $5 \mathrm{ng} / \mathrm{ml}$. Em 11 (25,0\%), as determinações obtidas do CEA sérico e do biliar foram menores ou igual a $5,0 \mathrm{ng} / \mathrm{ml}$.

Nos enfermos operados por carcinoma colo-retal, os valores do CEA biliar foram mais expressivos do que os determinados no sangue ( $p<0,0001)$ (Tabela 1). Já no grupo de doentes colecistectomizados, os valores do CEA biliar foram significativamente menores do que os do CEA sérico $(p=0,46)$ (Tabela 2). Os níveis de CEA biliar nos doentes com carcinoma colorretal foram significativamente maiores $(\mathrm{p}<0,0001)$ do que naqueles colecistectomizados. A relação (ratio) CEA biliar / CEA sérico nos doentes operados por carcinoma colorretal e nos enfermos colecistectomizados mostrou valores significativamente maiores no grupo de doentes com neoplasia do intestino grosso do que naqueles colecistectomizados ( $\mathrm{p}<$ 0,0001) (Tabela 3).

TABELA 3 - Médias dos valores da razão CEA biliar/sérico obtidos dos doentes operados por carcinoma colo-retal e por colelitíase.

\begin{tabular}{l|c|c|c|c|c}
\hline \multicolumn{1}{|c|}{ Variável } & Média & D.P. & Mínimo & Máximo & N \\
\hline $\begin{array}{l}\text { CEA biliar/ } \\
\text { sérico }\end{array}$ & $22,1 \mathrm{ng} / \mathrm{ml}$ & 48,5 & $0,02 \mathrm{ng} / \mathrm{ml}$ & $287,0 \mathrm{ng} / \mathrm{ml}$ & 44 \\
\hline $\begin{array}{l}\text { CEA biliar/ } \\
\text { sérico }\end{array}$ & $0,6 \mathrm{ng} / \mathrm{ml}$ & 0,3 & $0,3 \mathrm{ng} / \mathrm{ml}$ & $1,9 \mathrm{ng} / \mathrm{ml}$ & 10 \\
\hline
\end{tabular}

Legenda: Wilcoxon $(Z)=-1,992$ p $<0,46 *$ D.P.= Desvio padrão $\mathrm{N}=$ Número

Nesse estudo, não houve relação significativa entre os valores do CEA sérico $(\mathrm{p}=0.60)$ ou do CEA biliar $(\mathrm{p}=0,78)$ com a classificação de Dukes. Os valores do CEA sérico e biliar também não mostraram relação significativa com a localização das lesões neoplásicas no colo direito, colo esquerdo ou reto $(\mathrm{p}=0,93 \mathrm{e}$ $\mathrm{p}=0,53$, respectivamente). 
Quatro $(9,1 \%)$ doentes com carcinoma colorretal, operados com intenção curativa e cujos níveis de CEA biliar estavam elevados, apresentaram, durante sua evolução, metástases hepáticas diagnosticadas, inicialmente, pela elevação dos níveis séricos do CEA no pós-operatório, cuja média foi $8,6(0,3$ a 22,8) $\mathrm{ng} / \mathrm{ml}$. Somente dois deles apresentavam nível de CEA sérico aumentado no momento da operação, porém os quatro enfermos apresentaram nível de CEA biliar elevado, com média de 228,4 (6,6 a 571) ng/ml e correspondiam a $13,8 \%$ dos 29 doentes com CEA biliar elevado. Neles, as metástases hepáticas se desenvolveram, em média, cerca de 9,7 (3 a 17) meses após a retirada da lesão colo-retal primária. Três deles foram estadiados como Dukes $\mathrm{C}$ e um como Dukes B (Tabela 4). Exceto uma doente (Dukes B) que apresentou recidiva da doença com metástases hepáticas disseminadas em ambos os lobos, os outros três enfermos foram submetidos a hepatectomia para a retirada de suas metástases. Dois deles faleceram cerca de nove meses num caso e 21 meses noutro após a retirada das lesões hepáticas. Embora não tenha sido realizada necropsia nesses doentes, não havia evidência de recidiva da neoplasia até o momento dos óbitos. Um terceiro enfermo está vivo, sem doença ativa, cerca de oito meses após a extirpação das metástases hepáticas. Os restantes quarenta enfermos estão vivos, sem sinais de recidiva da doença, seis a 48 meses após retirada da lesão colorretal primária.

TABELA 4 - Local da lesão, estadiamento de Dukes e valores do CEA sérico e biliar, obtidos dos doentes operados por carcinoma colorretal com metástases hepáticas no seguimento.

\begin{tabular}{c|c|c|c|c}
\hline Caso & Local & Dukes & CEA sérico (ng/ml) & CEA biliar (ng/ml) \\
\hline 1 & Transverso & $\mathrm{B}$ & 22,8 & 571,0 \\
\hline 2 & Reto & $\mathrm{C}$ & 2,1 & 292,0 \\
\hline 3 & Reto & $\mathrm{C}$ & 9,4 & 44,1 \\
\hline 4 & Sigmóide & $\mathrm{C}$ & 0,3 & 6,6 \\
\hline Média & & & 8,6 & 228,4 \\
\hline
\end{tabular}

\section{Discussão}

A capacidade de prever o aparecimento de metástases hepáticas em doentes operados curativamente de carcinoma colorretal poderia influenciar o uso de quimioterapia adjuvante e intensificar o seguimento dos doentes com indicação de tratamento cirúrgico para lesões extirpáveis. ${ }^{18,20,24,25}$

A determinação do CEA biliar pode representar método potencialmente sensível para o diagnóstico de metástases hepáticas do carcinoma colo-retal, uma vez que lesões hepáticas menores que $1 \mathrm{~cm}^{3}$ podem produzir elevadas concentrações de CEA na bile. ${ }^{11,16,22,26}$

Huang \& Tang $^{27}$ estudaram o CEA sérico e o biliar obtido por drenagem com tubo duodenal no pré-operatório em doentes com afecção benigna e com carcinoma colorretal com e sem metástases hepáticas. Esses autores verificaram que a diferença entre os valores do CEA biliar nos doentes operados por carcinoma colo-retal com e sem metástases hepáticas foi significativa, mostrando que o nível de CEA biliar auxilia na confirmação das lesões hepáticas. Novell e col. ${ }^{19}$ e Moura e col. ${ }^{22}$ estudaram o nível de CEA biliar e sérico em doentes com carcinoma colorretal e sugeriram que a determinação do CEA biliar pode ser útil no diagnóstico de metástases hepáticas ocultas. Nos quatro doentes do presente estudo que evoluíram com metástases hepáticas e em 29 (65.9\%) outros enfermos que não apresentaram metástases, os valores do CEA biliar também foram significativamente maiores do que aqueles do CEA sérico. O seguimento médio de 16,5 meses não foi ainda suficiente para a avaliação conclusiva da determinação do CEA biliar como parâmetro preditivo do aparecimento de metástases hepáticas nesses doentes, pois o seguimento médio de, pelo menos, 60 meses aumentaria a possibilidade de recidiva hepática da doença e forneceria subsídios mais consistentes para a avaliação da utilidade do CEA biliar.

Nos doentes com metástases hepáticas do carcinoma colorretal, a capacidade concentradora da vesícula biliar foi apontada como o mecanismo responsável pela elevação dos níveis de CEA quando comparados aos níveis séricos. ${ }^{18,28,29} \mathrm{O}$ encontro de valores elevados de CEA biliar na ausência de metástases hepáticas também pode ser creditado ao fato do CEA biliar ser derivado não somente das metástases hepáticas, mas também do tumor primário. ${ }^{29}$ Esta situação contribuiria para a existência da relação direta entre os níveis de CEA sérico e biliar, ${ }^{13}$ isto é, quando os níveis séricos estão significativamente elevados, os níveis biliares também estarão, e portanto, os níveis de CEA produzidos pelo tumor primário podem elevar os níveis biliares, mesmo na ausência metástase hepática. Estes eventos poderiam justificar o achado da presente casuística, onde 25 (86,2\%) enfermos com CEA biliar elevado não apresentaram metástases hepáticas até a última consulta de seguimento. Entretanto, outros estudos ${ }^{13,14,17,30}$ sugeriram que os níveis aumentados de CEA na bile em presença de metástases hepáticas são produzidos, exclusivamente, pelas neoformações no fígado, não se originando da circulação portal, o que poderia indicar que os doentes com CEA biliar elevado possuem doença metastática hepática não diagnosticada. Paul e col. ${ }^{14}$ sugeriram que o CEA biliar prevê doença hepática oculta somente quando colhido após a remoção do tumor primário, o que evitaria contribuição significativa do CEA sérico sobre os níveis biliares. Resta comprovar se o encontro de níveis séricos biliares elevados de CEA durante a extirpação do carcinoma colorretal, como ocorreu com $27(61,4 \%)$ dos doentes desse estudo, constituiria critério de seleção para monitorização dos níveis de CEA biliar no pós-operatório. 
Yeatmane e col. ${ }^{11}$ encontraram níveis de CEA biliar elevados em $70 \%$ de doentes com carcinoma colorretal extirpado de maneira curativa, cuja ultra-sonografia intra-operatória hepática foi normal. No seguimento médio de 30 meses desse grupo de doentes, $13 \%$ deles apresentou metástases hepáticas, resultado próximo ao encontrado pelo presente estudo que constatou 9,1\% de metástases hepáticas com seguimento médio de 16,5 meses. Destri e col. ${ }^{28}$ encontraram acurácia diagnóstica do CEA biliar de $91 \%$ nos operados por carcinoma colorretal com ou sem metástases hepáticas e de 89,5\% em doentes que evoluíram com metástases hepáticas. Ishida e col. ${ }^{21}$ analisaram a relação dos valores CEA na bile da vesícula biliar colhida durante a operação e no sangue periférico com o aparecimento de metástases hepáticas metacrônicas do carcinoma colo-retal. Em 49 doentes sem evidências de metástases hepáticas no momento da operação, os níveis elevados de CEA biliar foram preditivos do aparecimento de metástases hepáticas metacrônicas com $75 \%$ de sensibilidade, $85 \%$ de especificidade e $84 \%$ de acurácia. Em outro estudo, Ishida e col. ${ }^{15}$ mostraram que doentes com CEA biliar ou ratio CEA biliar/ CEA sérico elevados podem ser candidatos à recidiva hepática.

No presente estudo, quatro $(9,1 \%)$ doentes operados curativamente de carcinoma colorretal desenvolveram metástases hepáticas após a retirada da lesão neoplásica. Como a média do tempo de seguimento dos doentes foi de 16,5 meses, é possível que o número de doentes acometidos por metástases hepáticas e CEA biliar elevado aumente com o prolongamento do seguimento, o que poderia tornar a determinação do CEA biliar parâmetro preditivo do desenvolvimento das lesões hepáticas.

Entretanto, outros estudos não compartilharam a idéia de que o CEA biliar possui valor preditivo em relação ao aparecimento de doença hepática metastástica. Dorrance e col. ${ }^{18}$ determinaram os níveis de CEA sérico e biliar de 26 doentes submetidos à cirurgia curativa e seguidos na média de tempo de 63,5 meses. Doze $(46,1 \%)$ doentes sobreviveram sem recidiva e $14(53,8 \%)$ deles faleceram por recidiva da neoplasia. $\mathrm{O}$ valor médio do CEA sérico no grupo livre da doença foi significativamente maior do que no grupo com recidiva. A acurácia do CEA sérico como índice preditivo de metástases hepáticas ocultas foi de $77 \%$ comparada com $72 \%$ para o CEA biliar, sem diferença significativa. Os autores concluíram que a determinação do CEA biliar intra-operatório não é mais acurada do que a do CEA sérico como índice preditivo da ocorrência de metástases em doentes submetidos à cirurgia potencialmente curativa do câncer colorretal. Panaguzzi e col. ${ }^{21}$ estudaram o seguimento de doentes operados por carcinoma colorretal sem metástases hepáticas porém com níveis de CEA biliar elevado e concluíram que, embora os níveis de CEA biliar estivessem elevados nos doentes com metástases hepáticas, esses níveis não representam parâmetro preditivo para a sua presença no carcinoma colorretal. Garcia e col ${ }^{18}$ determinaram a concentração de CEA biliar de 24 doentes com carcinoma colorretal, onde 21 enfermos não apresentavam evidências de metástases hepáticas, enquanto outros três eram portadores de lesões no fígado. Esses autores acompanharam os doentes, em média, por 32,3 meses. Três deles desenvolveram metástases hepáticas e todos exibiram
CEA biliar elevado. Os autores afirmaram não existir relação clara entre os valores de CEA biliar e o aparecimento de metástases hepáticas, embora reconheçam que a amostra não foi suficientemente ampla para conclusões mais definitivas.

Nesta casuística, nove $(20,5 \%)$ enfermos apresentaram valores do CEA biliar menores do que os respectivos valores de CEA sérico. Possível explicação para este fato seria a de que os mecanismos de depuração hepática do CEA produzido pela neoplasia colorretal primária podem não estar saturados e, conseqüentemente, os níveis excretados na bile seriam menores do que os séricos.

Poder-se-iam alargar as indicações da hepatectomia por metástases hepáticas e introduzir-se mais precocemente procedimentos quimioterápicos locais ou sistêmicos, cirurgia radio-imunoguiada, ou, ainda, tratamento com utilização de anticorpos monoclonais anti-CEA.

Para comprovar se os valores de CEA biliar são realmente seguros para detectar a recidiva hepática em estádio precoce são ainda necessários estudos prospectivos com tempo de seguimento adequado e com intervalos padronizados entre a extirpação da lesão neoplásica colorretal e a retirada de amostras biliares.

\section{Conclusão}

$\mathrm{O}$ antígeno carcinoembrionário (CEA) biliar surge como ajuda para identificar doentes com metástases hepáticas não diagnosticadas. Em enfermos com recidiva neoplásica constatada, a sensibilidade da determinação do CEA na bile é maior do que a dos valores encontrados no sangue.

\section{Referências}

1. Adson MA. Resection of liver metastases: when is it worthwhile? World J Surg 1987;11:511-20.

2. Kievit J, Bruinvels JD. Detection of recurrence after surgery for colorectal cancer. Eur J Cancer 1995;31A:1222-5.

3. Fantini GA, Decosse JJ. Surveillance strategies after resection of carcinoma of the colon and rectum. Surg Gynecol Obst 1990;171:26773.

4. Finlay IG, McArdle CS. Occult hepatic metastasis in colorectal carcinoma. Br J Surg 1986;73:732-5.

5. Stone MD, Kane RA, Bothe A, Jessup JM, Cady B, Steele GD. Intraoperative ultrasound imaging of the liver at the time of colorectal cancer resection. Arch Surg 1994;129:431-6.

6. Gold P, Freedman SO. Demonstration of tumor-specific antigens in human colonic carcinomata by immunological and absortion techniques. J Exp Med 1965;121:439-62.

7. Fletcher RH. Carcinoembryonic antigen. Ann Intern Med 1986:104: 66-73.

8. Hohenberger P, Schlag PM, Gerneth T, Herfarth C. Pre- and postoperative carcinoembryonic antigen determination in hepatic resection for colorectal metastases. Predictive value and implication for adjuvant treatment based on multivariate analysis. Ann Surg 1994:219:135- 43.

9. King J, Caplehorn JR, Ross WB, Morris DL. High serum carcinoembryonic antigen concentration in patients with colorectal liver metastases is associated with poor cell-mediated immunity, which is predictive of survival. Br J Surg 1997;84:1382-5. 
10. Bakalakos EA, Burak WE Jr, Young DC, Martin EW Jr. Is carcinoembryonic antigen useful in the follow-up management of patients with colorectal liver metastases? Am J Surg 1999;177:2-6.

11. Yeatman TJ, Bland KI, Copeland III EM, Hollenbec JI, Souba WW, Vogel SB, Kimura AK. Relationship between colorectal liver metastases and CEA levels in gallbladder bile. Ann Surg 1989;210:505-12.

12. Yeatman TJ, Kimura AK, Copeland III EM, Bland KI. Rapid analysis of carcinoembryonic antigen levels in gallbladder bile. Ann Surg 1991;213:113-7.

13. Paul MA, Viser JJ, Mulder C, Blomjuos JGAM, Van Kamp GJ, Cuesta MA, Meijer S. Detection of occult liver metastases by measurement of biliary carcinoembryonic antigen concentrations. Eur J Surg 1996;162:483-8.

14. Paul MA, Viser JJ, Mulder C, Van Kamp GJ, Cuesta MA, Meijer S. The use of biliary CEA measurements in the diagnosis of recurrent colorectal cancer. Eur J Surg Oncol 1997;23:419-23.

15. Ishida H, Hojo I, Gonda T, NakajimaH, Hirukawa H, Itoh M, Satoh $\mathrm{K}$, Higushi T, Toyooka M, Yoshinaga $\mathrm{K}$ et al. Measurement of bile CEA levels in patients with colorectal cancer: is it of value for diagnosis of occult liver metastases aiming at prophylatic regional hepatic chemotherapy? Gan To Kagaku Ryoho 1993;20:1551-4.

16. Paganuzzi M, Onetto M, de Paoli M, Castagnola M, de Salvo L, Civalleri d, Grossi CE. Carcinoembryonic antigen (CEA) in serum and bile of colorectal cancer patients with or without detectable liver metastases. Anticancer Res 1994;14:1409-12.

17. Huang M, Tang D, Li B. Evaluation of biliary CEA in the diagnosis of colorectal cancer with liver metastases. Zhonghua Zhong Liu Za Zhi 1999;21:45-7.

18. de Andre S, Garcia B, Madrona AP, Ayalla MP, Parrilla PP. Utilidad de la determinacion del antígeno carcinoembrionario em la bilis para la prediccion del desarollo metástasis hepáticas tras la resection de cancer colorrectal. Med Clin (Barc) 1997;108:396.

19. Novell F, Trias M, Molina R, Filella X. Detection of occult liver metastases in colorectal cancer by measurement of biliary carcinoembryonic antigen. Anticancer Res 1997;17;2743-6.

20. Dorrance HR, McGregor JR, McAllister, Path MRC, O'Dwyer PJ. Bile carcinoembryonic antigen levels and occult hepatic metastases from colorectal cancer. Dis Colon Rectum 2000;43:1292-6.
21. Ishida H, Yoshinaga K, Gonda T, Ando M, Hojo I, Fukunari H, Iwama T, Mishima Y. Biliary carcinoembryonic antigen levels can predict metachronous liver metastasis of colorectal cancer. Anticancer Res 2000;20:523-6.

22. Moura RM, Matos D, Galvão Filho MM, D'ippolito G, Sjzenfeld J, Giuliano LM. Value of CEA level determination in gallbladder bile in the diagnosis of liver metastasis secondary to colorectal adenocarcinoma. Sao Paulo Med J 2001;119: 110-3.

23. Li Destri L, Curreri R, Lanterin R, Gagliano G, Rodolico M, Di Cataldo A, Puleo S. Biliary carcinoembryonic antigen in the diagnosis of occult hepatic metastases from colorectal cancer. J Surg Oncol 2002;81:8-11.

24. Wang JY, Chiang JM, Jeng LB, Changchien CR, Chen JS, Hsu KC Resection of liver metastases from colorectal cancer: are there any truly significant clinical prognosticators? Dis Colon Rectum 1996:39:847-51.

25. Gervaz P, Blanchard A, Pampallona S, Mach JP, Fontolliet C, Gillet M. Prognostic value of postoperative carcinoembryonic antigen concentration and extent of invasion of resection margins after hepatic resection for colorectal metastases. Eur J Surg 2000:166:557-61.

26. Uchino R, Kanemitsu K, Obayashi H, Hiraoka T, Miyauchi Y. Carcinoembryonic antigen (CEA) in the bile of patients with biliary diseases. Am J Surg 1994;167:306-8.

27. Frikart L, Rounier K, Match J-P, Givel JCI. Potential value of biliary CEA assay in early detection of colorectal adenocarcinoma liver metastases. Eur J Surg Oncol 1995;21:276-9.

28. Svenberg T, Hammarstrom S, Hedin A. Purification and properties of biliary glycoprotein I (BGPI). Immunochemical relationship to carcinoembryonic antigen. Mol Immunol 1979;16:245-52.

29. Thomas P. Studies on the mechanisms of biliary excretion of circulating glucoproteins. Biochem J 1980;192:837-43.

30. Tabuchi Y, Deguchi H, Imanishi K, Saitho Y. Comparison of carcinoembryonic antigen levels between portal and peripheral blood in patients with colorectal cancer. Cancer 1987;59:1283-8.

Polycarpo A, Topciu FR, Neto JZA, Lopes LS, Catelani LGC, Zerwes MHT, Gonçalves S, Waisberg J. Carcinoembryonic antigen (CEA) determination in detection of hepatic metastasis from colorectal carcinoma . Acta Cir Bras [online] 2003; 18 Supl 4. Available in www.scielo.br/acb.

ABSTRACT - Purpose: Carcinoembryonic antigen (CEA) determination in gallbladder bile was used in a prospective study concerning the morphological and clinical features of the neoplasm and the occurrence of hepatic metastasis. Methods: CEA levels in the gallbladder and peripheral blood were studied in 44 patients with colorectal carcinoma and 10 patients with uncomplicated cholelithiasis from samples collected immediately before extirpating the colorectal neoplasms or cholecystectomy (values of up to $5 \mathrm{ng} / \mathrm{ml}$ were considered normal). Results: In the 44 patients with colorectal carcinoma who underwent operation with curative intent, the average level of serum CEA was $8.5 \mathrm{ng} / \mathrm{ml}$ and for bile CEA, $74.5 \mathrm{ng} / \mathrm{ml}$. In the patients with uncomplicated cholelithiasis who underwent cholecystectomy, the average level of serum CEA was $1.9 \mathrm{ng} / \mathrm{ml}$ and for bile CEA, $1.2 \mathrm{ng} / \mathrm{ml}$. Four patients who underwent extirpation of the colorectal carcinoma without evidence of hepatic metastasis and with an average bile CEA value of $213.2 \mathrm{ng} / \mathrm{ml}$ presented hepatic metastasis between three and seventeen months after removal of the neoplasms. Conclusion: The high CEA levels in gallbladders of patients undergoing curative operation for colorectal carcinoma may indicate the presence of hepatic metastasis. Such patients must be followed up with special attention to the diagnosis of such lesions.

KEY WORDS - Carcinoembryonic antigen. Colorectal neoplasms. Neoplasm metastasis.

Correspondência:

Rua Aparecida, 130 apto 32

09751-330 São Bernardo do Campo - SP 